\title{
The Research of Intelligent Parking System based on Internet of Things Technology
}

\author{
Meng Li \\ Shanghai University of \\ Engineering Science \\ Songjiang Shanghai \\ 201620, China
}

\author{
Chen Deng \\ Shanghai University of \\ Engineering Science \\ Songjiang Shanghai \\ 201620, China
}

\author{
Weimin Zhu \\ Shanghai University of \\ Engineering Science \\ Songjiang Shanghai \\ 201620, China
}

\begin{abstract}
With the improvement of people's living standards, Large and medium-sized cities and some small and medium cities in the demand for private cars is becoming more and more big. This comes with increasing demand for parking spaces. Traditional fixed parking spaces and surface parking spaces have been unable to meet the growing demand for parking spaces, on the other hand, the bustling city commercial district, government agencies and offices and other places will inevitably appear parking difficult problem. At the same time, the growth of parking fees in some areas has brought a lot of economic burden to the office workers. Parking is difficult, the impact is not limited to the parking itself, which brings a series of urban management issues. Through the investigation and interview path discovery, staggered time sharing spaces is an effective means to solve the problem of parking.
\end{abstract}

For the current parking is difficult, parking is not convenient to find parking spaces. To ease traffic congestion, save fuel to reduce exhaust emissions for the purpose, this paper proposes an efficient smart car system, including mobile terminal service system, data acquisition system, data transmission system and dynamic guidance system. System through a realtime network released vacant parking information and are equipped with GPS dynamic map navigation, when a vehicle enters the strange parking lot, system will enable indoor RFID tag localization, intelligent vehicle guidance function, also have the lock network control and carpooling bit line trading function.

\section{General Terms}

Realtime Parking

\section{Keywords}

Mobile terminal; RFID; dynamic guidance; online transaction

\section{INTRODUCTION}

1998 American Vice President Al Gore proposed the concept of "Digital Earth", thus, the concept it triggered is applied to the movement of social informatization "digital city", The national mapping Geographic Information Bureau has promoted the construction and popularization of the Digital City Geospatial Framework in the whole country. 2008, IBM president and CEO Peng Mingsheng put forward the concept of the wisdom of the earth, causing the world's countries and regions to build smart city building boom.

Real time parking (real-time parking) is an emerging concept in recent years, it is a kind of quick find vacant parking intelligent parking system, when the driver is near the parking lot, he or she can obtain real-time parking information. According to the development trend of the smart city Gartner released, real-time parking is in the rising and rapid development period, in the next 5 to 10 years it will reach the peak period. It is achieved by the mobile device of driver to detect parking spaces, without any special facilities or external sensors, so compared to the existing technology, it is more economical.

\section{DOMESTIC AND FOREIGN RESEARCH SITUATION}

\subsection{Domestic Research Status}

Study on the parking problems in cities of China began in the 80 s of the last century, in recent years, due to the cars of the rapid growth and urban parking facilities is not perfect, the parking problem has become an important factor restricting the development of urban communications, many domestic scholars and transportation workers on the theory of urban parking facilities were in-depth study, including parking demand forecast analysis, parking location analysis, parking lot design, parking guidance information system.

\subsection{Foreign Research Status}

In foreign countries, the research on the urban parking problem earlier began in the middle of the last century, in the United States, Germany and some of the early industrialized countries, with the recovery of the economy, more and more people began to have a car. In these countries, people begin to pay attention to the city parking problem, and thus they began in-depth study.

In the intelligent traffic management system, intelligent transportation research work can trace back to the 1960-1970, ERGS electronic route guidance system (Electronic Route Guidance System) developed at that time, intelligent transportation system (ITS) concept in 1990 was put forward by the Intelligent Transportation Society (IVHS America) and was vigorously promoted in the world.

\section{MARKET ANALYSIS}

\subsection{Main Problems at Present}

1. The area of private parking spaces in the working days (Friday to Monday) almost in zero application state, resulting in a huge waste of resources.

2. Only part of the business district of parking field is labeled into the map marker range, small and medium-sized effective parking is not timely updated into the map, the owners is difficult to find parking space near the destination field, even knowing the parking position, but the owner can't be accurately informed of the of the parking spaces vacancy information, this would lead to blind search. 
3. Most of the existing parking using artificial management, this need to be equipped with a large number of full-time management personnel to carry out vehicle parking guidance, and this will increase the cost of parking management.

4. Parking into the parking field can not be quickly drive into the parking position of the vehicle, parking can only be in the presence of disorderly flow to find vacant spaces. It not only occupies a floor the white lane and lead to loss of vehicle fuel, but even cause traffic congestion.

5. After parking into a strange car park parking lot, usually owners forget the position of the car, which has brought about the inconvenience and other issues.

\subsection{Solutions}

According to the above problems, as well as the development of parking industry in developed countries, the parking problem has spawned to a huge parking management market and parking management strategy market.

At present, a number of new parking guidance systems and parking facilities are emerging to solve the above problems., but most of them are parking guidance and parking facilities renovation, it does not fundamentally solve fewer parking spaces, Rebuilding the parking facilities, such as the construction of large underground garage will bring huge cost.

In order to improve the free parking utilization rate and parking information, intelligent management level, and to the owners provide a more environmentally friendly and more human parking experience, hereby developed the "wisdom spell parking spaces, wonderful benefit life" online to find parking spaces, which is a one-stop booking spaces, GPS map navigation, parking lot local map precision navigation and positioning, lock control, carpool system. The system solves the difficult troubles of office workers, cross regional office staff to look for parking space, Adopt the method of " timesharing lease " to use private parking spaces reasonably, with online registration information and transaction, system can release the parking information and find parking spaces and reduce parking management cost, and it relatively increases the available parking space. At the same time, it can maximally reduce vehicles bypass resulting in energy waste and emissions, the greatest degree of reach the purpose of energy saving and emission reduction.

\section{SCHEME DESIGN}

The system includes car line of real name registration information, vacant parking information release, and parking for parking information search, recommend the destination path, indoor RFID tag positioning and navigation, lock control module. Parking publisher, according to their own parking spaces occupied needs in real time, released vacant time through the mobile terminal device, when there is parking demand, through the mobile terminal equipment intelligent positioning search, so as to establish the online booking relationship with the parking lot. According to the parking spaces, parking spaces can be found in the location of the parking lot. Through the mobile terminal equipment to complete the information recognition function to enter the residential access control, if the system is shown to have an appointment with the user, you can enter the cell.After entering the area, the system opens the indoor positioning and navigation, parking can grasp their own position in real time, and then the parking enter the parking space in accordance with the district's intelligent system. When the vehicle completes the parking, the parking space immediately opens the lock function, until the completion of the parking lot after the completion of the parking process to confirm that the lock system automatically unlock. In a day, the number of private parking spaces can be released in accordance with the transaction information for many times. The overall structure of the system is shown in figure 4.1.

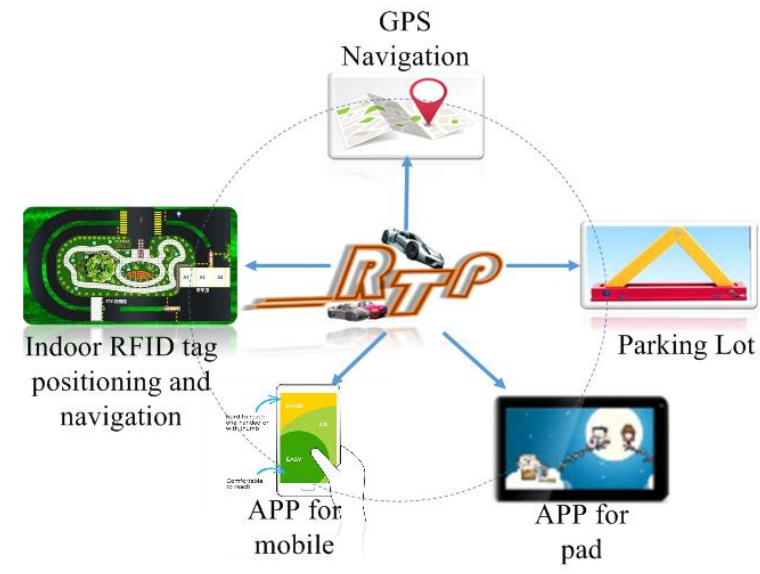

Figure 4.1 Overall structure diagram of the system

\subsection{Hardware design}

\subsubsection{Sandbox hardware}

The design of the vehicle indoor positioning, residential intelligent parking induction and lock function is realized fully by the sand table platform of intelligent transportation system.

The sandbox uses the commercial gateway as the main controller, and each unit module is connected by 485 bus. The unit sub module is controlled by the STM32 as the controller, which can receive gateway control commands and control the corresponding hardware devices. Each functional sub module can be composed of multiple unit modules. The sandbox hardware structure is shown in figure 4.2. 


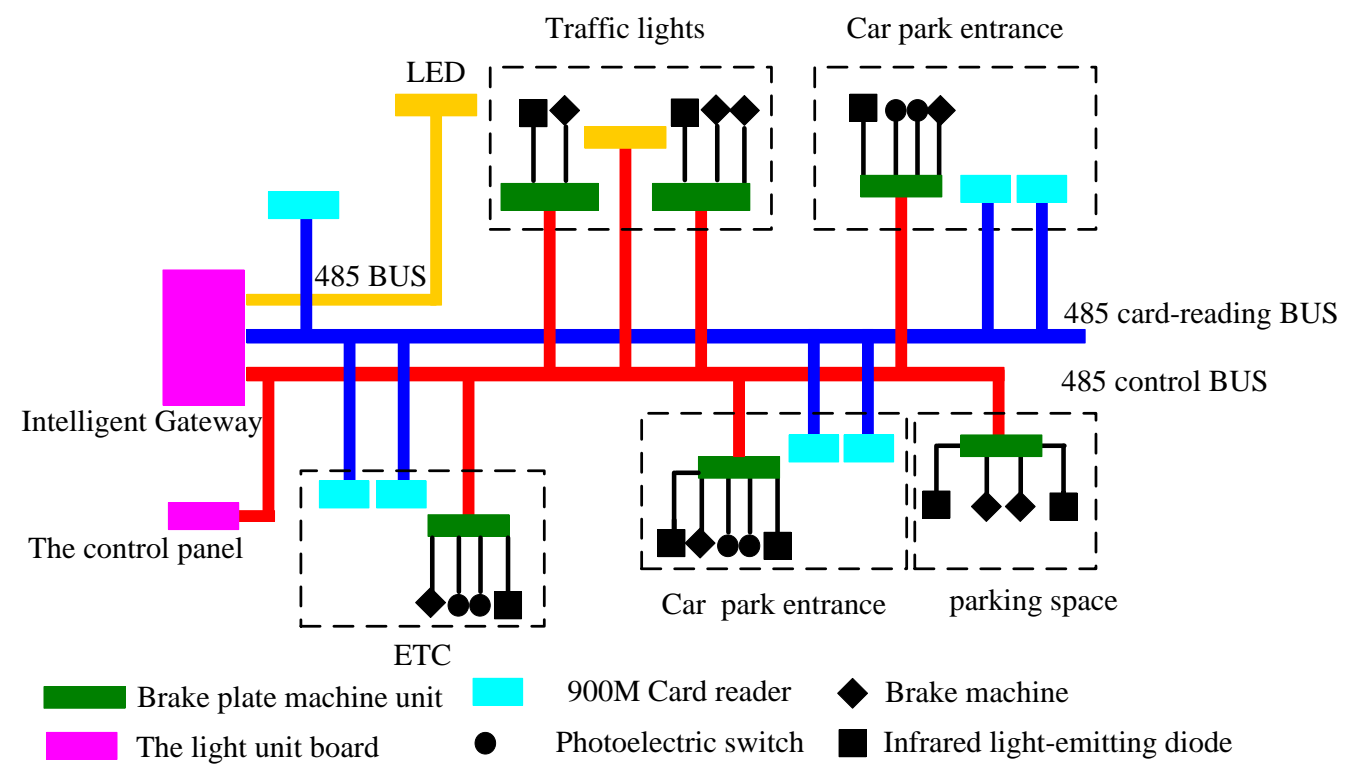

Figure 4.2 Table hardware structure chart

Intelligent traffic sand table design to three lanes, the smart car can run simultaneously in different lanes. Among them, the design of the A lane and B lane has ETC brake and parking lot. Traffic lights can be achieved in all lanes, in addition, in the tunnel entrance design of the $\mathrm{C}$ lane, the reader is used to read the vehicle information through multiple

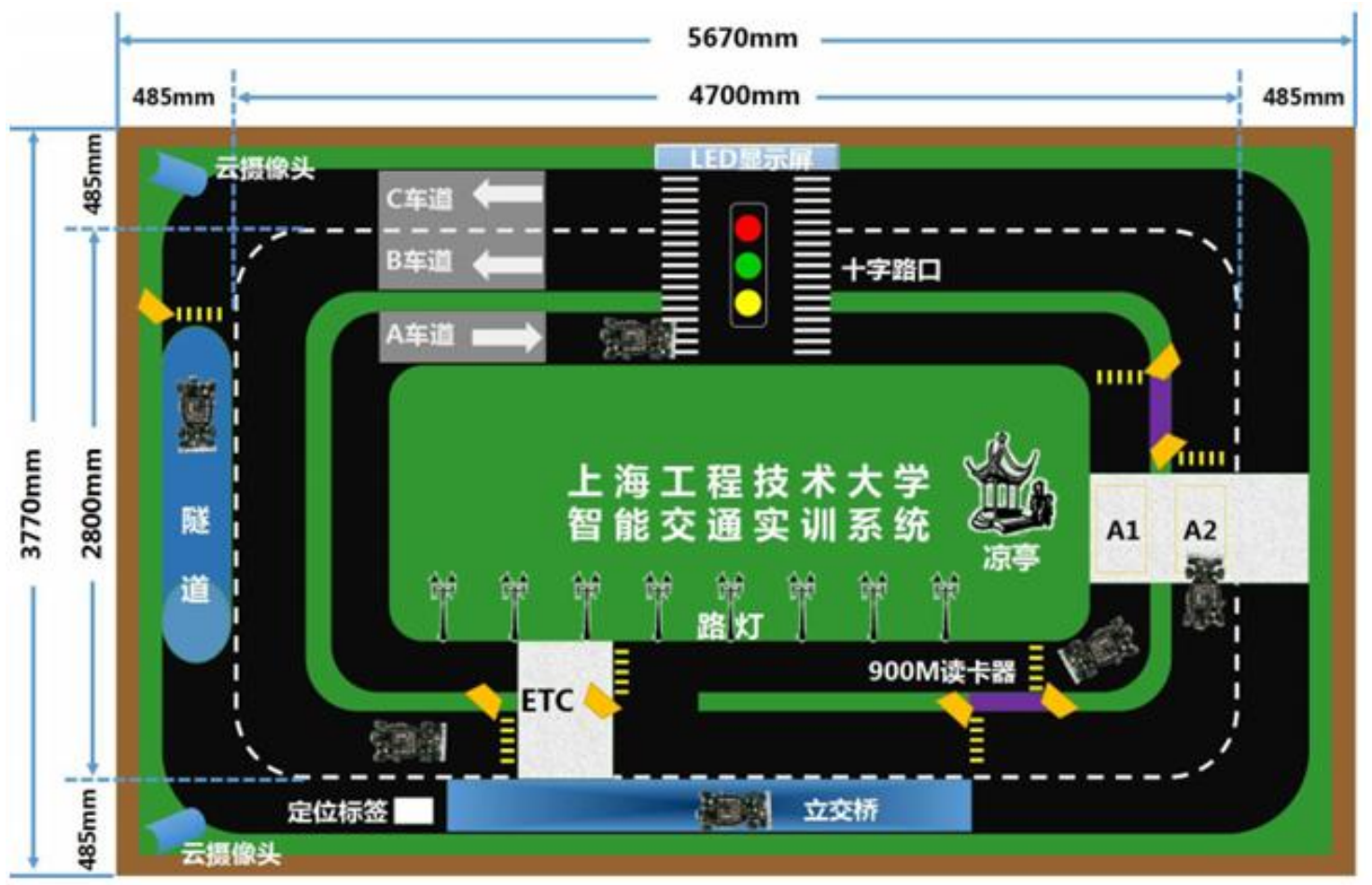

tunnels. The sand table is equipped with two cloud camera for real-time monitoring and ETC road crossroads. A lane and B lane are the full function lane, In the $\mathrm{C}$ lane, there is a tunnel and overpass. The main table panel size are $5670 \mathrm{~mm} * 3770 \mathrm{~mm} * 800 \mathrm{~mm}$. Its main panel is shown in figure 4.3 .

Figure 4.3 the main table panel

\subsection{Software design}

System software part is mainly composed of users' information registration, customer information management, vacant parking information release, real-time parking information query, GPS navigation, indoor intelligent parking guidance, parking reservation and delay, online payment and lock control. Coordination between multiple parts to ensure the implementation of the whole system, and the block execution is conducive to maintenance.

The design of the system is mainly based on the LabVIEW platform, which successfully transplanted the interface into a portable device for operation and simulation. Combining with 
the intelligent traffic sand table and the existing hardware device, the simulation of the release parking, searching, parking, route guidance and the others has realized successful.

\subsubsection{Introduction of the function module}

(1) Personal information registration and management

The related information of private owner needs to be certificated before using the client. Only when private owners pass the real-name authentication can they get the client information released by the relevant authority. Certified by real-name registration is also in order to strengthen community garage management, prevent the potential or other security hidden danger.

\section{(2) Vacant parking information release}

The users can upload their vacant parking time and their address to the system server, then the system will summarize the parking information and classify it and send it to the users.
Information releasing end is the core part of the whole system, it's also the start of the system operation. It mainly completes the information release, send the effective information to the Internet in the process of operation, so the users can get effective parking information timely and accurately.

(3) Real-time query parking space information

There are two ways provided to the users when they want to quarry the parking information. One is just to search the parking lot by locking the user's current location. The other way is to have a self-service search via the toolbar provided by the system. After the search, the system will automatically match user's request and provide various choice, then the user can choose it based on their own requirements. Of course, the system will sort the all parking address based on the distance between the destination and the current location, then give the optimal recommended path to the users. The operation process of real-time query parking information as shown in figure 4.4 .

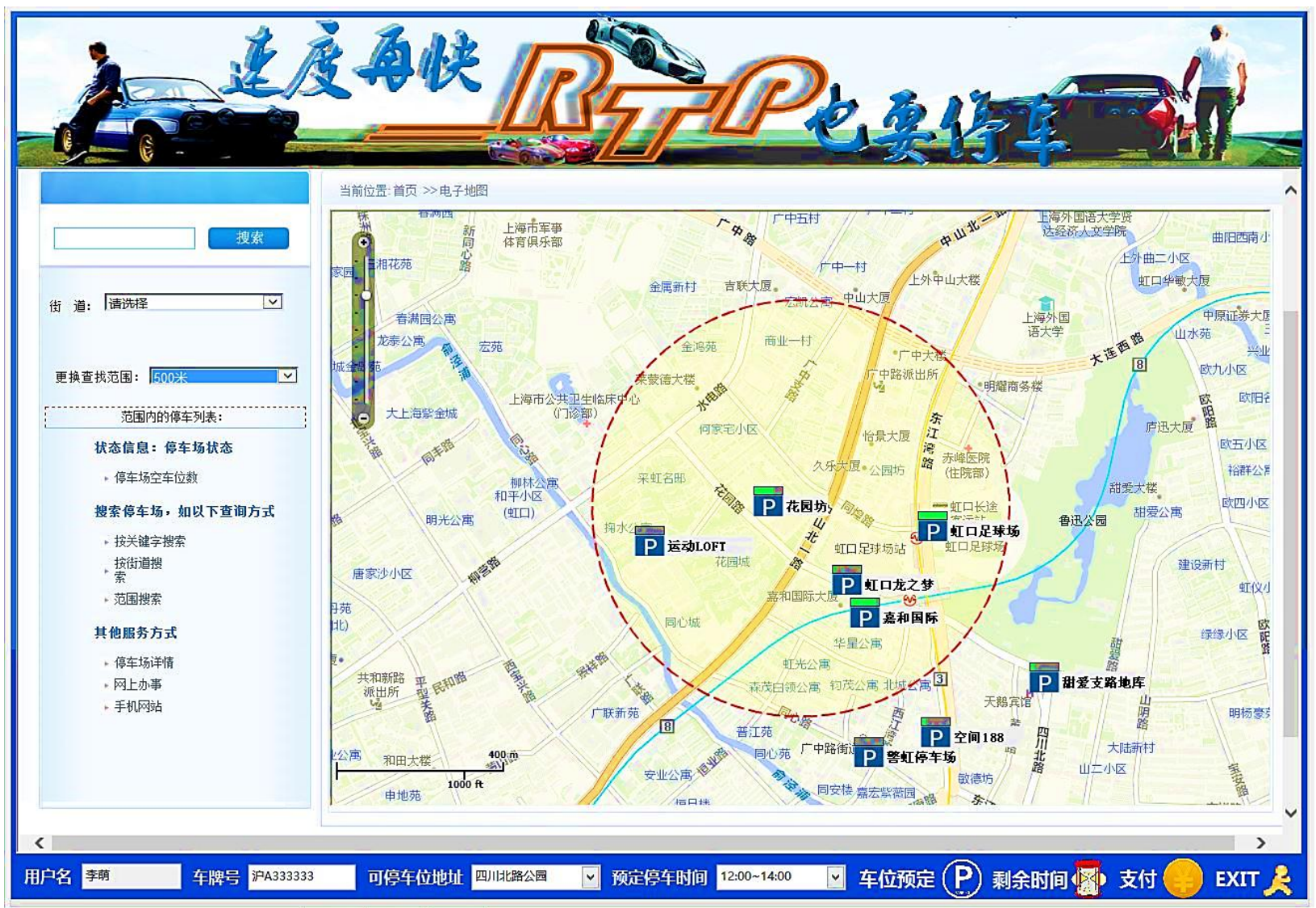

Fig4.4 real-time query parking information

(4) Parking reservation

Based on the information recommended by the system, the user can determine the proper time to park. Once they clicked on the confirmation, the system will automatically complete the parking space booking, and reminds the user of successfully booking.

\section{(5) GPS navigation}

At present most of the private cars has been equipped with GPS vehicle navigation, flexible application of GPS technology to the client device which can bring convenience for parking searching purposes. GPS navigation technology is not the focus of this design part, in this system we realized the connection to the map through the web access technology, most importantly, users can accomplish the GPS mobile navigation and location according to their requirements as shown in figure 4.5 . 


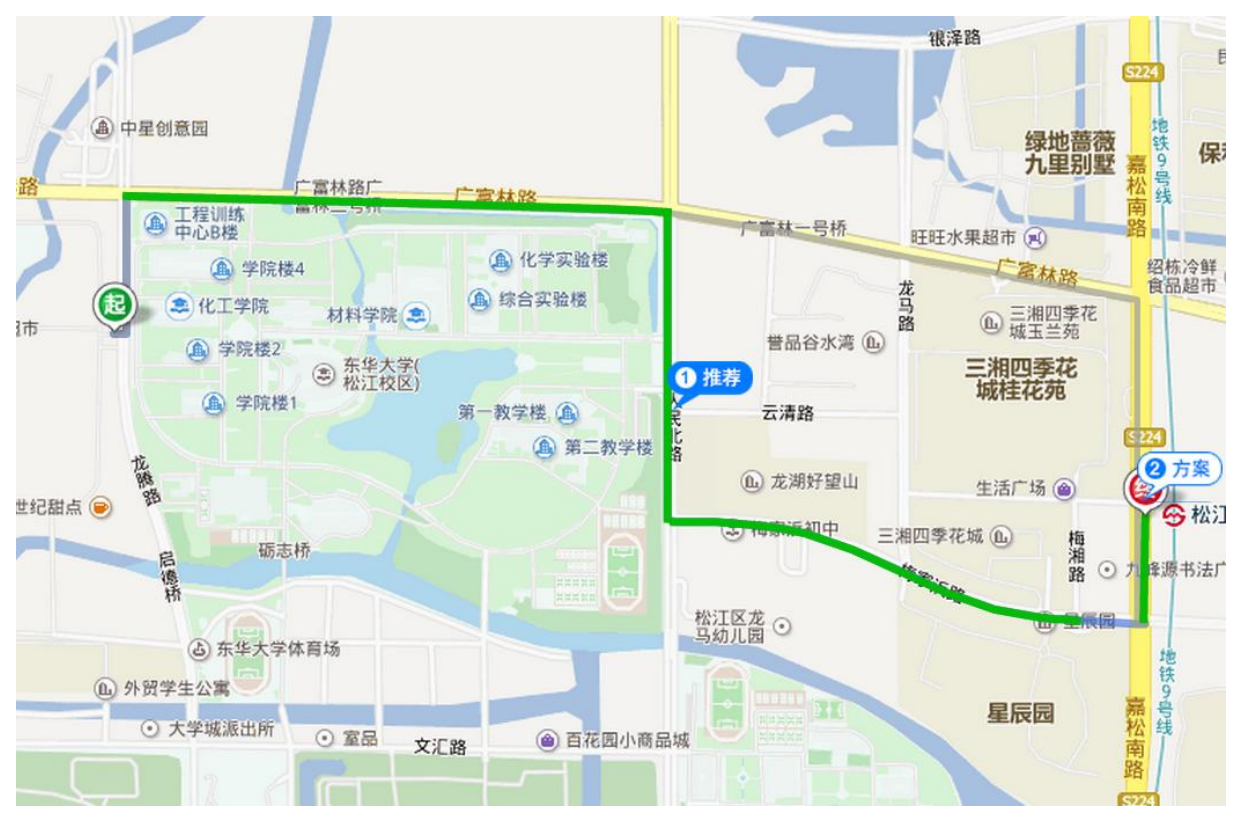

Fig4.5 GPS navigation

(6) intelligent parking guidance

Considering the structure of parking area is complex, and the parking space distribution are scattered over the various place. The first problem a driver is up against is that it's difficult for him to find his parking point especially when he was new here. Secondly when he want to take his car he may forget the parking point which may annoy the driver. By the configuration of RFID tag equipotential system, private cars can be real-time access to their location within the community, then timely find the parking location. Once entering into the community, the user can open mobile terminals on the local map, the system will automatically provide good path planning for the user, so that the user can move to the parking space as soon as possible. The intelligent parking guidance interface as shown in figure 4.6.

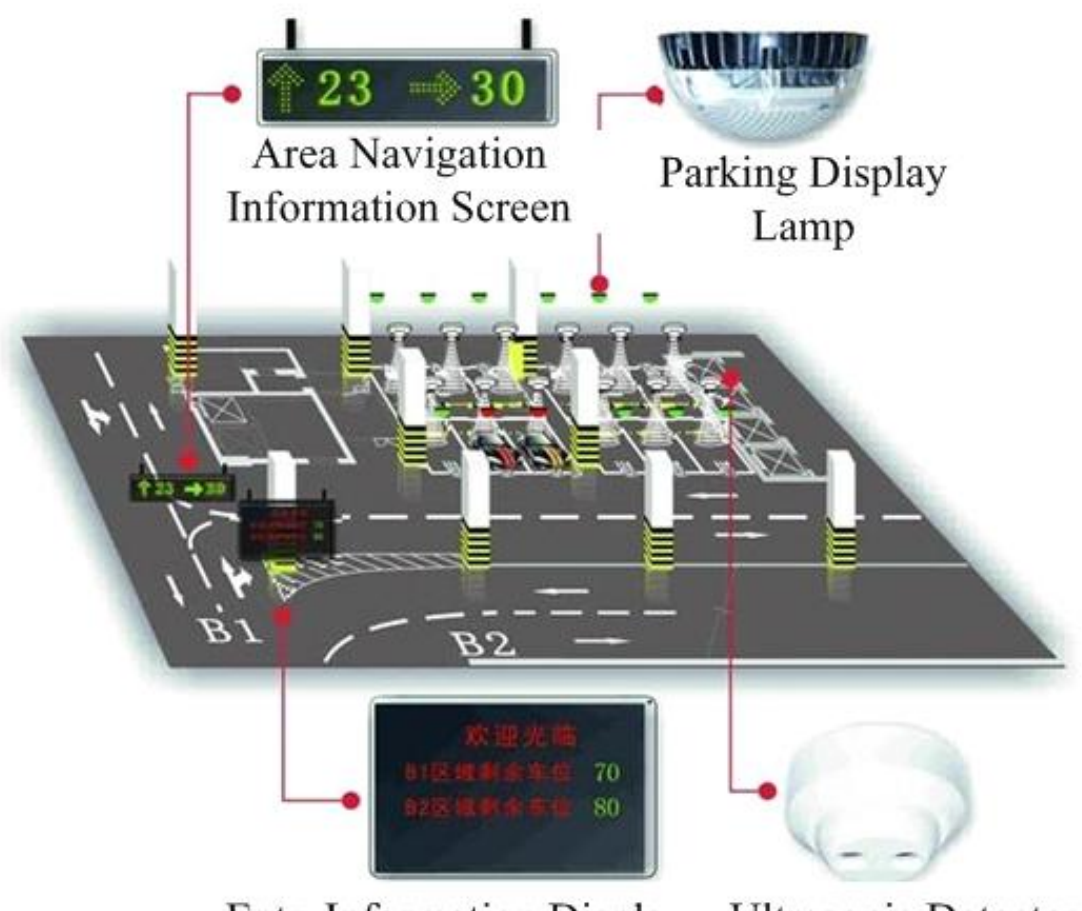

EntryInformation Display Ultrasonic Detector

Fig4.6 intelligent parking guidance interface

(7) Parking time query and delay

The user not only can view the parking information timely through the mobile terminal, but also can extend the parking time. Which can reduce the additional charges due to the stop time is too long. 
(8) Online payment

Online payment function is mainly used for user to pay the parking fee and open the parking lock. The lock only can be open after the users pay the money successfully, otherwise it will remains closed and locked. In order to ensure the transaction between the publishers and the users accomplished effectively, the system has built the parking networking lock protection. At the same time the system protect both the user and the publisher's legal rights and interests.

\section{CONCLUSION}

This system is used to increase the effective use of the free parking, at present it's mainly suitable for the release of information of a private cars and online transactions. Otherwise the indoor small scale intelligent positioning guidance system which is based on RFID can be widely used in large shopping malls, hospitals, shopping square and so on.

And now the design of the construction of the parking lot has been toward to the large scale, so accurate parking route guidance system will like GPS positioning technology is widely used. With the popularity of mobile phone APP function, carpool technology based on mobile terminal equipment will be widely recognized and used later.

\section{ACKNOWLEDGE}

This work is supported by Postgraduate Student Research Innovation Project Funds of Shanghai University of Engineering Science Grant NO.E1-0903-15-01029.

\section{REFERENCES}

[1] Wang Shaohua,Lu hao. Key Techniques of Intelligent Transportation System[J]. Surveying and Mapping and Spatial Geographic Information. 2013, 36: 88-91.

[2] Liang Jun,Huang Sai. Development Opportunities and Challenges from the Digital City to the Wisdom City[J]. Geographic Information World.2013.11(1):81-86

[3] Xia Ying. Spatio-temporal Data Analysis of Key Techniques in Intelligent Transportation System Study[J]. Chong qin. Southwest university.2012

[4] China heavy Machinery Industry Association Parking Equipment Management Committee. Mechanical
Parking Equipment[M].Bei jing: Ocean press.2005:10186

[5] Shladover.S.E. Progressive Deployment Steps Leading Toward an Automated Highway System (AHS)[C]. Transportation Research Board. Wshington DC, 2000: 154-161.

[6] Kikuchi H , Kawasaki S , Nakazato G. ITS in Japan:Current Status and Future Directions[C]. 7th World Congress on Intelligent TransportSystems.Turin. Italy, 2000: 3149-3152.

[7] Bishop R. Intelligent Vehicle Applications Worldwide[J]. Intelligent Systems and their Applications, IEEE, 2000,15(1): 78-81.

[8] Ching-Hung Lee, Yu-Hui Wang, Amy J.C. Trappey, Service design for intelligent parking based on theory of inventive problem solving and service blueprint[J]. Advanced Engineering Informatics, In Press, Corrected Proof, Available online 18 November 2014.

[9] Masoud Honarmand, Alireza Zakariazadeh, Optimal scheduling of electric vehicles in an intelligent parking lot considering vehicle-to-grid concept and battery condition[J]. Energy, Volume 65, 1 February 2014, Pages 572-579.

[10] David H.S. Lima, Andre L.L. Aquino, OASys: An opportunistic and agile system to detect free onstreet parking using intelligent boards embedded in surveillance cameras[J]. Journal of Network and Computer Applications, Volume 46, November 2014, Pages 241-249.

[11] Nadav Levy, Marc Render, Itzhak Benenson, Spatially explicit modeling of parking search as a tool for urban parking facilities and policy assessment[J]. Transport Policy, Volume 39, April 2015, Pages 9-20

[12] Masoud Honarmand, Alireza Zakariazadeh, Shahram Jadid, Integrated scheduling of renewable generation and electric vehicles parking lot in a smart microgrid[J]. Energy Conversion and Management, Volume 86, October 2014, Pages 745-755. 